\title{
Incorporating Practice Theory in Sub-Profile Models for Short Term Aggregated Residential Load Forecasting
}

\author{
Bruce Stephen, Senior Member, IEEE, Xiaoqing Tang, Poppy R. Harvey, \\ Stuart Galloway, and Kyle I. Jennett
}

\begin{abstract}
Aspirations of grid independence could be achieved by residential power systems connected only to small highly variable loads if overall demand on the network can be accurately anticipated. Absence of the diversity found on networks with larger load cohorts or consistent industrial customers makes such overall load profiles difficult to anticipate on even a short term basis. Here, existing forecasting techniques are employed alongside enhanced classification/clustering models in proposed methods for forecasting demand in a bottom up manner. A Markov chain based sampling technique derived from practice theory of human behavior is proposed as a means of providing a forecast with low computational effort and reduced historical data requirements. The modeling approach proposed does not require seasonal adjustments or environmental data. Forecast and actual demand for a cohort of residential loads over a five-month period are used to evaluate a number of models, as well as demonstrate a significant performance improvement if utilized in an ensemble forecast.
\end{abstract}

Index Terms-Human factors, load modeling, power systems, practice theory, renewable generation.

\section{INTRODUCTION}

$\mathbf{L}$ EGACY power systems featuring large and geographically distributed load bases connected to centralized generation can exploit routine and climatic diversity to smooth out their demand profiles. Even without predictable large industrial and commercial loads, residential loads are present in sufficient numbers that some system operators assign them a single load profile on the assumption that the large cohort will average out the diversity [1]. Small power systems, possibly in rural areas, with diverse housing stock face a greater challenge in evaluating their anticipated demand making grid independence a less viable proposition without maintaining prohibitively expensive reserve margins. Behavioral changes driven by consumer routine and building stock variation [2]

Manuscript received April 17, 2015; revised July 10, 2015 and September 4, 2015; accepted October 17, 2015. Paper no. TSG-00434-2015.

B. Stephen is with the Advanced Electrical Systems Research Group, Institute of Energy and Environment, University of Strathclyde, Glasgow G1 1RD, U.K. (e-mail: bruce.stephen@strath.ac.uk).

X. Tang, P. R. Harvey, S. Galloway, and K. I. Jennett are with the Department of Electronic and Electrical Engineering, University of Strathclyde, Glasgow G1 1XW, U.K.

Color versions of one or more of the figures in this paper are available online at http://ieeexplore.ieee.org.

Digital Object Identifier 10.1109/TSG.2015.2493205

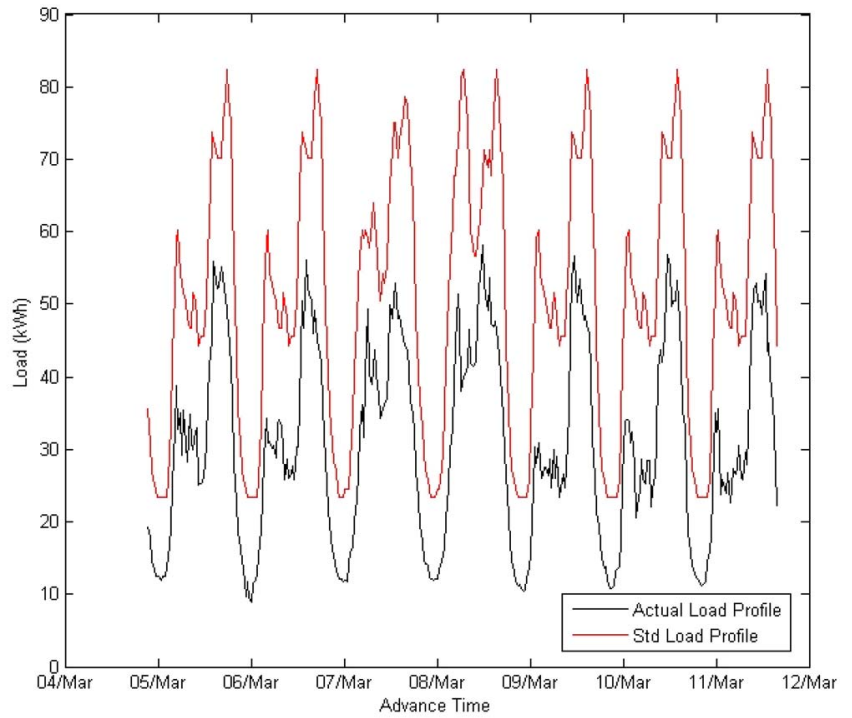

Fig. 1. Residential feeder metered load profile compared against a scaling of the U.K. standard residential load profile [1] over a single week.

result in abrupt temporal changes consistent with a statistically complex distributed process, which rules out regression-based forecasting techniques [1] that can be employed at higher levels of load aggregation such as the primary substation.

The nature of this problem can be better understood if the underlying residential loads are considered in context. Pursuing the approach typically adopted at national levels, e.g., [1] whereby a single load profile is assumed to merely increase by a factor of the number of loads, offers insufficient accuracy as Fig. 1 demonstrates: on a small cohort of 123 residential customers, the aggregated load fails to match the U.K. domestic load profile class scaled up accordingly. The absence of diversity increases shoulder period variability and can result in load voids at both peak and trough periods. Although a key problem is the overestimate of peaks and troughs this is an understandable overestimate at a national level to accommodate generation margins but a potential problem for islanded microgrids that require foreknowledge of the local load to absorb or supplement the renewable generation resource.

The challenge presented by this data is three-fold: firstly, residential loads lack the predictable patterns that may be 
used to forecast future behavior using conventional stationary regression time series models; secondly, while environmental data is often incorporated into load forecast models at the regional and national level [2], [3], highly localized data is rarely available and thirdly, historical load data is almost nonexistent for specific residential and low voltage customer loads so seasonally adjusted predictions are not possible in most cases. The models employed in this paper are used to forecast an aggregated load profile on a day-ahead basis, based on short term generalizations of consumption derived from a reduced number of very recent constituent half hourly electrical load measurements taken at the premises incomer. Neither seasonal nor daily correction/standardization is applied as the model learns only from data supplied weeks or days prior to the forecast, nor are additional parameters are required to be measured (such as humidity or ambient temperature) and subsequent forecasts rely only on the load profile observed on the previous day. The aggregated load on the network feeder is then forecast by aggregating the forecasts from all constituent premise loads on the network to evaluate generation requirements in a bottom up manner. The advantage of aggregating the forecasts in this way is that it takes into account the constituent behaviors whereas a single forecast would not include the details of this inherent variability.

This paper is organized as follows: the next section reviews load forecasting techniques previously used in power systems operation and planning - many of these techniques have produced highly accurate forecasts but on at the higher voltage levels of the network where diversity produces smoother and more consistent load profiles. Section III reviews the reasons that motivate different models for smaller power systems and the models that can accommodate them. Section IV proposes extensions to this methodology and draws justification for this from the field of Practice Theory in Human Factors analysis. Section V shows how these models, inherently stochastic, can be sampled to provide forecasts of load on a 24 hour ahead (the typical definition of short term load forecasts) basis. Section VI utilizes a metered residential premises data set to demonstrate forecasting performance for the proposed model and the most prominently used models in the field: Persistent forecasting, Feed Forward Neural Networks and Autoregressive models. Performance metrics are also discussed as the dynamics of this particular data set can render the outputs of some traditional error measures misleading. Section VI compares the performance of the 5 models and comments on their relative strengths. Section VII concludes with potential areas of model improvement and an analysis of where forecasting presents the greatest challenges in the context of power systems.

\section{Short TERM LOAD ForecAsting}

Load forecasting techniques for 24 hours in advance, commonly referred to as 'short term', are well established for the zonal/HV/MV and national scale networks, e.g., [4]-[6]. Two frequently used approaches to short term load forecasting are Autoregressive (AR) type models [4], [5] such as
Autoregressive Moving Average (ARMA) and Autoregressive Integrated Moving Average (ARIMA); and also Neural Network (NN) based approaches [6], [7]. AR models utilize recurring patterns in time series data that imply a linear relation between time points and use these to predict a specified number of time points into the future. The number of time points considered is denoted as the order of the model, so a model that uses the previous time point to predict the next is a first order model, one that uses two time points is a second order model and so on. The NN models are essentially universal approximators to an arbitrary relation between input and output data shown to the model: in the load forecasting application, the output data would be the time points of the forecast period while the inputs may be the corresponding load at the current days' time points. For example, in [8] a NN approach was used to forecast loads one day in advance on a 230 node feeder/network. This produced 24 hourly load estimates as its output from an input of the previous days load observations coupled with weather forecasts and day indicators. This model was learned over 210 weekdays and then tested on 30 weekdays - weekends were not considered. Reference [8] also employed AR/ARMA/ARIMA models utilizing 6 weeks of data; ARMA and ARIMA models are estimated on a previous weeks basis ( 5 days over 24 hours leads to a model order of 120). As might be expected, the progressively more complex models yielded higher accuracy. Reference [8] noted the varying accuracies of load forecasting achieved at the various levels of the power system: $13.8 \%$ Mean absolute percentage error (MAPE) at village level, 5.15\% at University Campus level compared with a $1.97 \%$ best at national level, although it should be remembered that a 'campus' case is akin to a commercial load and is susceptible to business hours base loads that will reduce the challenge of forecasting. Reference [9] highlighted the more specific case of a number of residential feeder scenarios with forecast MAPE of between 10-35\%.

\section{Sub-PROFILED RESIDENTIAL LOADS}

References [9]-[11] note the greater levels of unpredictability exhibited by microgrids and sensitivity to end usage. The assumptions of weekend/weekday distinction, outlined by the uniform load profiling approach, are clearly not always valid and similar assumptions of weekday homogeneity are similarly invalid. References [5], [8], and [13] identified this and omitted weekends from their forecast model, placing focus entirely on weekdays. Several previous works have proposed the notion of representing individual loads using sub-profiles learned through clustering type algorithms. Exemplifying the difference between weekend and weekday behavior, [13] maps and then automatically classifies load pattern clusters as weekend and weekdays such is the difference between weekend and weekday behavior. Routine often dictates behavior [11] rather than the day of the week and this has motivated works such as [14]-[16] to first find generalizations of load profiles and forecast from this starting point. Weekend and public holidays can invoke changes in routine that manifests itself as different load profiles. Because of these shifts in behavior, there are resulting shifts in distribution which leads to the 


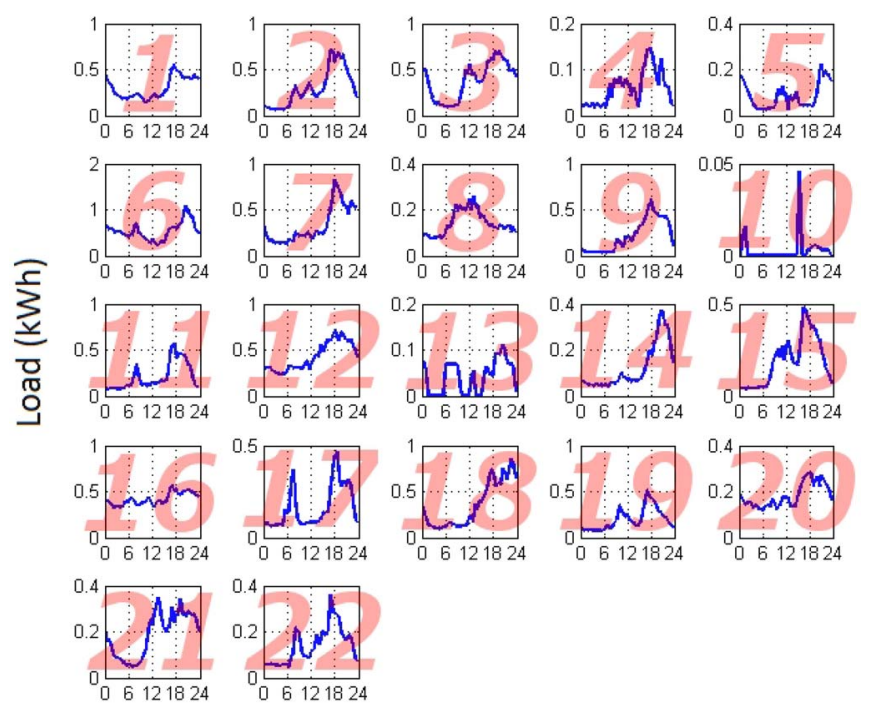

Time Step (hours)

Fig. 2. Optimal January model -22 half hourly load profiles recovered as Gaussian means; scale is in $\mathrm{kWh}$.

advance data exhibiting what is termed as non-stationarity, thus motivating the choice of cluster type models to generalize load before predicting what it will do next. One simple approach to generalizing load profiles is to look at it on a timestep by timestep basis - [17] presents an aggregated LV model of several feeders that is considered with a probability distribution generated for each time step in the same vein as [18], where loads are stratified. Reference [14] and [16] both use Self Organizing Maps (SOM) to identify recurring behaviors in daily load profiles.

To achieve a similar objective [18], [19] used mixtures of univariate and multivariate Gaussians respectively for modeling electrical load advances at various times of day but in a manner that still preserved the load profile within the model in an intuitive manner. Reference [19] demonstrated how this could be used to recover a number of recurring load profiles as the mean parameters of a Gaussian mixture model. For a randomly chosen subset of 30 houses over 30 days, Fig. 2 demonstrates the 22 profiles that recurred prominently. A key problem with using mixture models is estimating the model cardinality or the number of mixtures needed to best approximate the implied probability density of the non-stationary residential cohort load profile. Cardinality of the mixture model is dependent on data; model selection involves training a range of differently parameterized models and selecting one based on a predetermined criteria. Patterns will inevitably repeat over the course of the year - some load profiles observed in unoccupied properties (or possibly properties with good thermal insulation) will be observed irrespective of the time of year or outside conditions. The changes in behavioral diversity invoked by seasonal change will result in certain behaviors not being observed in all months. The 22 profiles recovered from the 900 (30 customers over 30 days) and shown in Fig. 3 were from a deemed optimal model selected by the formal criteria specified in [19].
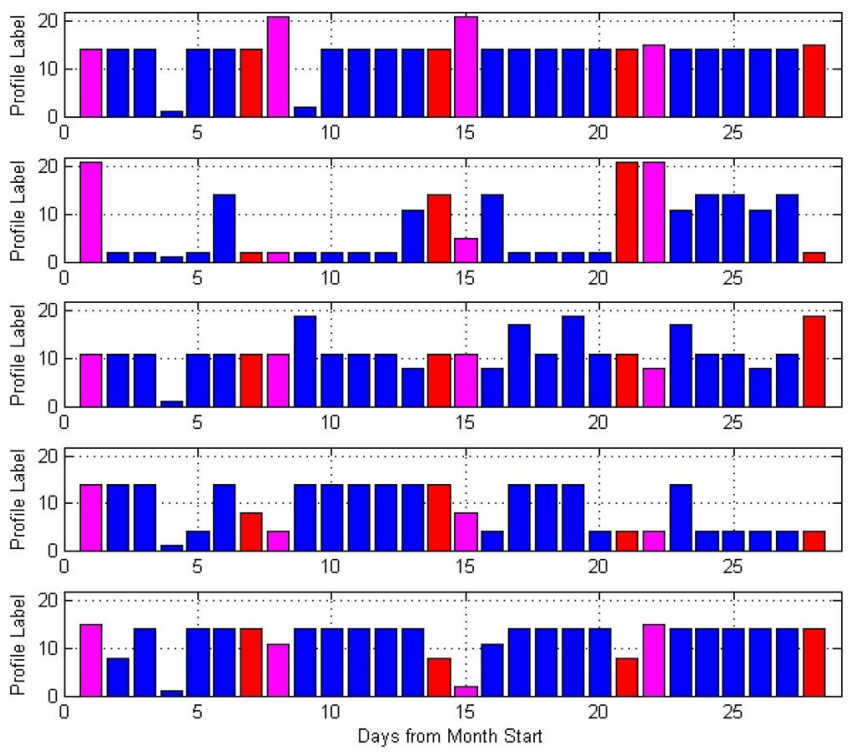

Fig. 3. Profile labels for five customers over the period of a single month -blue bars represent weekdays and red and pink bars represent Saturdays and Sundays respectively.
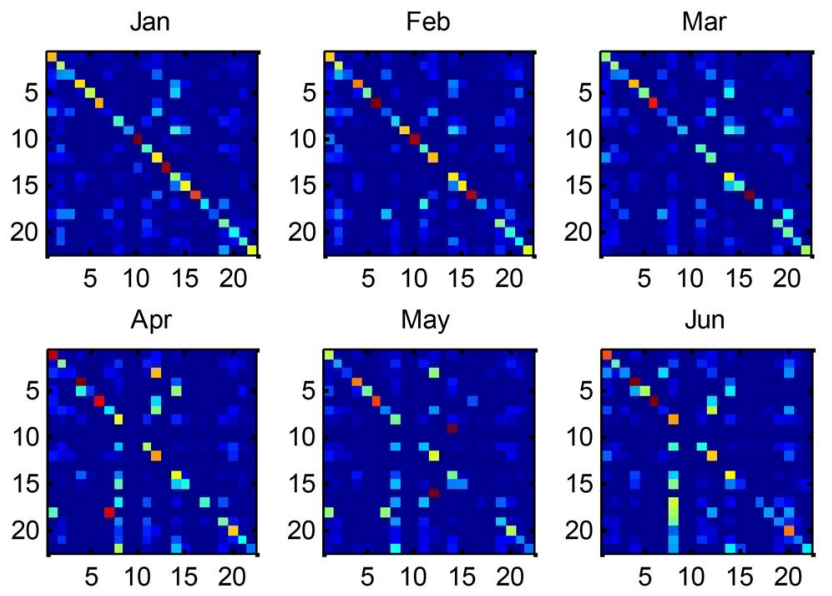

Fig. 4. Day to day movement between profile labels for each month encoded as transition matrices: rows represent labels; columns represent labels for the same premises on the following day. The color of each cell represents the frequency of a label occurring on a given day: blue represents infrequent through to red as most frequent.

Using a trained optimal mixture model on a set of meter data results in the load profiles being replaced with daily labels as would be possible with the models proposed in [14]-[17]. Fig. 4 shows the 22 profile model from Fig. 3 being applied to 5 households over a 1 month period. Day $t$ is given a label $c$ according to the load profile $l$ observed:

$$
c_{t}=\max _{c} \frac{\pi_{c} N\left(l_{t} ; \mu_{c}, \Sigma_{c}\right)}{\sum_{m=1}^{22} \pi_{m} N\left(l_{t} ; \mu_{m}, \Sigma_{m}\right)}
$$

A mixture model has scaling parameter $\pi$, along with a mean $\mu$ and covariance $\Sigma$ for each Gaussian component. Each day is consequently given a label of between 1 and 22 thus potentially reducing the problem of forecasting, broadly, to one of selecting the most likely next label in the sequence. 


\section{TRACKIng Changes In DEMAND}

As Fig. 3 indicates, the occurrence of a particular load profile is not completely random. The change and stability characteristics of energy usage behavior are dependent on various factors arising from personal and established routines which must be captured through additional model parameters. Extending the approach of [14], [15], and [19] to find the subpopulations, the conditional distribution of the profile labels given a particular day and also the transitions probabilities between profile labels, could also be estimated.

Justification for such modeling design decisions may be derived from the application of Practice Theory [20] to energy usage; [21] noted that the use of technology, in the form of appliances in the home contributed to overall energy usage in an ensemble effect through 'practices' - a 'practice' being a "temporally evolving, open ended set of doings and sayings...". These 'doings and sayings' (actually performative and communicative acts associated with executing daily routine) are identified as constituting hierarchical constructs along with tasks and projects that are executed 'occasionally, rarely or novelly'. The constraints and lower level cognitive predicates with which practices are assembled were identified by [21]: Practical Understanding, Rules, Teleoaffective Structures and General Understandings. These can be understood in the context of energy use as follows: 'Practical Understanding' constitutes not the activity but the underlying knowledge that governs its execution, that is, what to do, how to do it (e.g., the ordering of tasks) how to identify and react accordingly to cues or outcomes - examples may include wet appliance usage in domestic laundry tasks. 'Rules' indicate the basic constraints governing the technical or regulatory limits of the system, e.g., the rating of a particular appliance or the maximum/minimum duration it can be usefully operated. 'Teleoaffective structures' capture the goal oriented behaviors driven by 'normative moods or views' - these in turn may result from short term circumstance, e.g., boiling a kettle. From a longer term perspective, [2] noted on the subject of 'Habitus', the persistent patterns of thought perception and action, which could be driven by commuting habits and is therefore influenced by location which results in a consistently repeated behavior - charging an electric vehicle or use of wet appliances [22]. By the same reasoning, 'General Understandings' are shared beliefs possibly oriented by religious or ethical concern, may have a greater degree of persistence over time and occur at a regular interval or on a given day. Drawing on the behavioral influence of culture group and the routine driven nature of practice supports the notion of sub-profile load profiles - these practices will be repeated (albeit with noise and temporal variation) but will essentially play out on a regular basis resulting in a sequence of related dispersed practices that will manifest as transitions between particular daily load profile shapes. Much of this reasoning was implicitly drawn on in [23] to synthesize domestic load profiles from aggregating routine behavior patterns in appliance usage, themselves derived from survey participant responses.

Load profile classification approaches such as the mixture model approach of [19] allows each day to be retrospectively
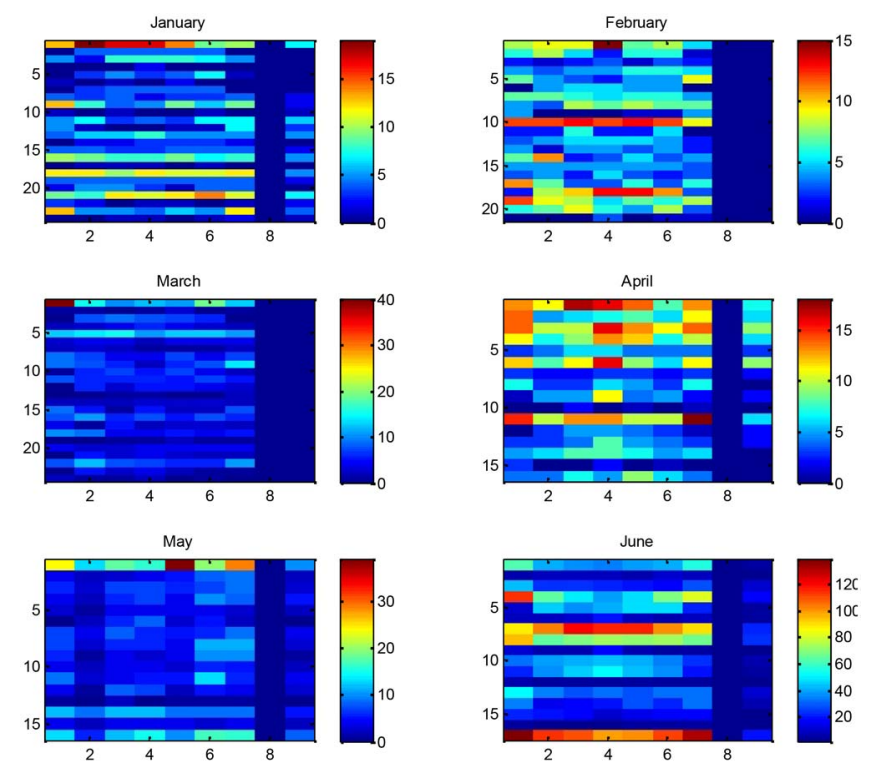

Fig. 5. Probabilistic relation between day of week (columns) and load profile label occurrence (rows) - the color of each cell represents the frequency of a label occurring on a given day: blue represents infrequent through to red as most frequent.

(at the completion of a single days' metering) assigned a label representing the model load profile that best represents the days load. This reduces a series of continuous valued load measurements to a much shorter sequence of discrete labels as Fig. 3 demonstrates.

By looking at the temporal variation in profile label along a sequence for a given premises, a matrix of transition probabilities, that embody the state space behaviors implied by [22] can be formed. Fig. 4 shows the transition matrices for a single optimal model produced from a 6 individual months' worth of data, demonstrating that the consecutive occurrence of particular load patterns does not remain the same from month to month. Day to day transition probabilities can be calculated for label $c$ as:

$$
P\left(c_{t}, c_{t+1}\right)=A_{i=c_{t} j=c_{t+1}}
$$

Particular behaviors will also be dictated by days of week as [13] noted. The probabilities for profile labels conditioned on weekdays $\left(d_{t}\right)$ are given by a normalized count of their occurrence:

$$
P\left(c_{t} \mid d_{t}\right)=W_{i=x_{t t}, j=d_{t t}}
$$

As with the transition probabilities shown in Fig. 4, recalculating these on a monthly basis for the same model shows how habits differ between months, akin to the findings of [24]. Fig. 5 shows this behavior evolving as a year progresses. Some load profiles have a high probability of occurring on a particular day - winter public holidays for example feature more pronounced behaviors than spring ones suggesting a spread of different behaviors as opposed to a 'staying in' behavior associated with the colder period.

However, across all premises monitored, both Fig. 4 and Fig. 5 demonstrate, that there are frequently recurring trends in transitions and day conditional load profile behaviors as the 
red colored cells indicate. In summary, extensions to the classification model of [19] to permit electrical demand forecasting, would consist of the following elements:

- An $m$-component Gaussian Mixture in $d$-dimensions where $d$ are the number of measurements over a single day and $m$ are the number of recurring profiles observed, selected by a theoretically valid model selection criteria such as [25]. This model will encode $m$ load profiles as an $m$ element set of $d$ dimensional mean vectors and $d$ by $d$ covariance matrices. The mixture components for the model, indicative of the probability a sub profile has of occurring, are represented by an $m$ dimensional vector $\pi$.

- A $m$ by 9 conditional probability matrix $W$, of the likelihood of seeing a given load profile $m$ on a given day of the week, a holiday or an extraordinary day.

- A $m$ by $m$ transition matrix $A$, denoting the probability of moving from one load profile to another on consecutive days.

The probability of any profile label being observed given a day of the week and a profile label from the previous day is given as follows

$$
P\left(c_{t+1} \mid c_{t}, d_{t+1}\right)=P\left(c_{t+1} \mid d_{t+1}\right) P\left(c_{t+1}, c_{t}\right)
$$

With a model in place for capturing recurring behaviors and how they occur over time, the next stage of development is to use the model to forecast how these behaviors recur on a day-ahead basis.

\section{A. Learning Day Ahead Demand Model for Residential Loads}

For a given, but not extensive, set of loads from individual premises over a short time period, an algorithm for the training of a load model would proceed as follows:

1. For metering data with $d$ daily advances, organize data from $N$ days and $M$ meters into $N$ by $M$ $d$-dimensional records of load. Initialize and train a mixture model on this d-dimensional historical load data and use this to evaluate the model order and therefore the form of the model distributions in the same manner as [19]. Initialization may be carried out by using randomly selected daily load profiles as the initial mixture means or estimating initial values using k-Means clustering.

2. From the same exemplar data, create $M$ sequences of d-dimensional load ordered by date.

3. Utilizing the trained model, replace the d-dimensional load profiles in each of the M sequences with a label representing the generalized profile that best approximates it as illustrated in Fig. 3.

4. Sum the label transition counts along each sequence and then normalize to obtain matrix $\mathrm{A}$ - the transition probability matrix (2).

5. Sum the label occurrences against each day of the week and then normalize to obtain day conditional matrix W (3).

6. Replace the model by repeating from step 1 at the end of each month to account for seasonal change.

\section{B. Day Ahead Demand Forecasting for Residential Loads}

For a trained model, a load profile forecast on a day ahead basis for any metered premises can then be conducted as follows:

1. If there is no previous days use, sample from a multinomial distribution using the mixture weights from the optimal model;

$$
c_{t}=\hat{c}_{t} \sim \operatorname{Multinomial}(\cdot \mid \pi)
$$

Which yields an estimate of the most likely load profile label $c$ at time $t$.

2. Otherwise:

a. Use the actual profile label for the previous days load profile $l$ obtained from the mixture model:

$$
c_{t}=\max _{c} \frac{\pi_{c} N\left(l_{t} ; \mu_{c}, \Sigma_{c}\right)}{\sum_{m=1}^{M} \pi_{m} N\left(l_{t} ; \mu_{m}, \Sigma_{m}\right)}
$$

b. Sample from the transition matrix row associated with the current day label to get the next day's label

$$
\hat{c}_{t+1} \sim \operatorname{Multinomial}\left(P\left(d_{t}=\text { tomorrow } \mid \cdot\right)\right)
$$

c. Sample from the day type matrix to get the most likely label for the given day of week

$$
\hat{c}_{t+1} \sim \operatorname{Multinomial}\left(P\left(\cdot \mid x_{t}=c_{t}\right)\right)
$$

d. Using the forecast label, select the Gaussian distribution in the profile model that is associated with it

3. Using the most likely mixture component - draw samples from the Gaussian distribution associated with it

$$
\hat{l}_{t+1} \sim N\left(\mu_{\hat{c}_{t+1}}, \Sigma_{\hat{c}_{t+1}}\right)
$$

4. At the end of the monitoring day, use the observed load profile to correct the label for the day using (7)

\section{Supporting Models}

This section outlines the alternative or possibly complementary forecasting techniques to the proposed method. All are expected to be trained on the same set of data and forecast over the same period and for the same time horizon.

\section{A. Flat Forecast}

A flat forecast is obtained in the manner of [26] by averaging over the load of the previous week and employing this as a forecast regardless of the time of day for all premises.

\section{B. Persistent Forecast}

A persistent forecast employing only the previous weeks advances averaged over a test data set and then employed at the corresponding time of day in the manner of [26]. 

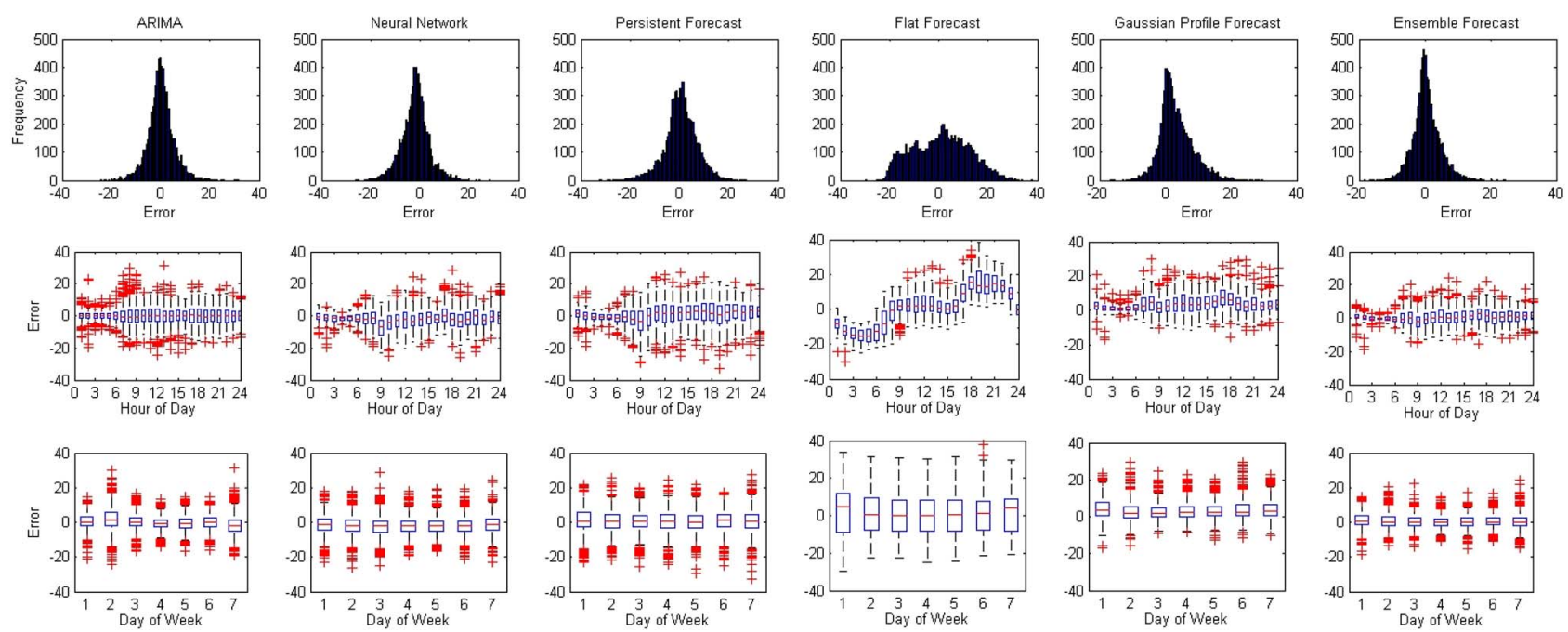

Fig. 6. Distribution of forecast MAE for all 5 models over the entire test period with disaggregation by hour and weekday. The rightmost plots represent the ensemble forecast encompassing all models except for the flat forecast.

\section{Feed Forward Neural Network}

A feed-forward Neural Network of the same design as used in [8] is employed; that is, one with 49 input nodes and 48 output nodes: one input for each half hourly time step plus an input for the day of week indicator. A forecast is made based on the previous days load observations as input. Different from the approach used by [8] is that all days of the week are forecast for, not just weekdays.

\section{ARIMA Model}

An ARIMA load forecast model was selected over the simpler AR and ARMA models to accommodate the known non-stationarity in the data. This is designed with a lag of 48 samples as in [8], and like this design, features distinct models for forecasting weekend and weekday behaviors. As with the Neural Network model, the forecast is made on the basis of the previous days load for each premises.

\section{Residential Forecasting Performance}

The loads used were taken from a 6 month January to June period. In many ways, this test network presents a challenging forecasting case - these are all drawn from real U.K. housing stock and feature very little homogeneity in terms of construction materials; occupant backgrounds/demographics are similarly diverse. The absence of any supporting data set, such as environmental measurements, means that this is an entirely load data driven model, emphasizing that the approach to electrical load forecasting advocated here is agnostic to demographic, building construction, environmental conditions and seasonal effects. The models are all learned from random $25 \%$ subsets of premises load data from a month previously and then tested on all customers from the subsequent month (e.g., train on January and forecast for February). Forecasts with all models are made at midnight every day for subsequent 48 half hourly increments. Load forecasts across all 123 premises on the network are aggregated together and compared to the actual aggregate load.
TABLE I

Comparison of Performance Forecast Over 5 Month Period

\begin{tabular}{cccc}
\hline \hline & $\begin{array}{c}\text { MAE } \\
(\mathrm{kWh})\end{array}$ & $\begin{array}{c}\text { MAPE } \\
(\%)\end{array}$ & $\begin{array}{c}\text { Optimal Adjusted } \\
\text { 4-Norm }\end{array}$ \\
\hline Persistent Forecast & 4.89 & 18.56 & 16560 \\
Flat Forecast & 9.43 & 44.89 & 17535 \\
ARIMA & 3.8 & 14.98 & 14667 \\
Neural Network & 4.38 & 17.65 & 15455 \\
Gaussian Load Profile & 4.22 & 14.69 & 13076 \\
Ensemble Forecast (all) & 3 & 11.13 & 12942 \\
Ensemble Forecast (no AR) & 3.3 & 12.19 & 13103 \\
Ensemble Forecast (no G) & 3.17 & 12.32 & 13526 \\
Ensemble Forecast (no N) & 3.21 & 11.63 & 13165 \\
& & & \\
\hline
\end{tabular}

\section{A. Error Metrics}

Error metrics used are the Mean Absolute Error (MAE), the Mean Absolute Percentage Error (MAPE) as well as the Permutated 4-Norm proposed in [26]. MAE is a standard measure for predictive efficacy while MAPE gives an impression of the order of magnitude of the forecast error [5]; the 4-Norm has been chosen to accommodate temporal shift in observed behaviors that may be attributable to delays in domestic routine.

Table I shows the overall performance comparison for the five short term load forecast models plus ensembles of various combinations. All models were implemented in Matlab and run on an Intel Quad Core i7 at 3.8 GHz with 16GB RAM.

Fig. 6 shows how each model performs over the entire testing period. Notable from Fig. 6 is the performance at morning peak times: the ARIMA models exhibit high errors whereas the Neural Network, the Gaussian approach and the persistent forecast have much lower errors at these times. This situation reverses for evening peaks, with the ARIMA model banding errors more tightly. Errors for all but the Gaussian forecast model are symmetric - the Gaussian model tends to underestimate load, reflected in the number of positive errors 


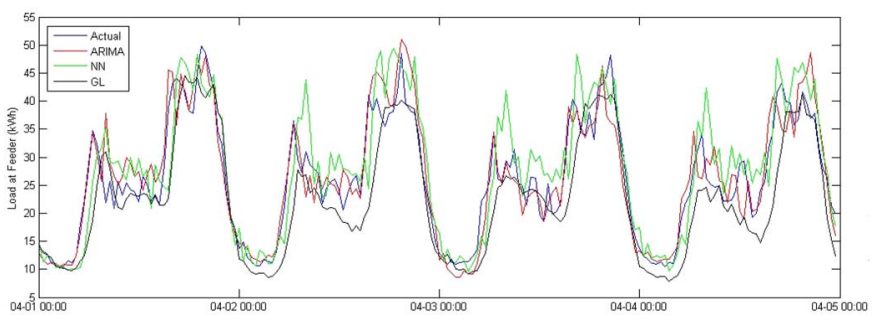

Fig. 7. Forecast and actual aggregated load comparison for the ARIMA, neural network and Gaussian load profile.

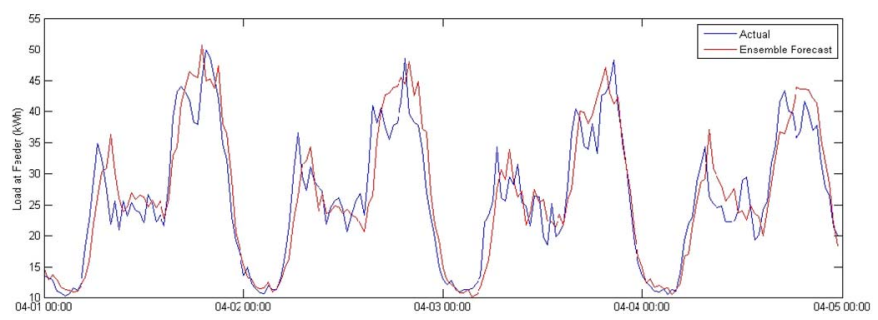

Fig. 8. Ensemble forecast and actual aggregated load comparison.

TABLE II

Comparison of Model Computation Time

\begin{tabular}{cc}
\hline \hline MODEL & $\begin{array}{c}\text { CPU Time } \\
\text { (seconds) }\end{array}$ \\
\hline Persistent Forecast & 0.180 \\
Flat Forecast & 0.187 \\
ARIMA & 406.6 \\
Neural Network & 2.216 \\
Gaussian Load Profile & 3.586 \\
\hline
\end{tabular}

in the histogram. The Neural Network overestimates with a pronouncedly reduced tendency for extreme underestimates.

Clearly, as with more predictable demands, different models have different strengths and weaknesses according to the dynamics exhibited at a given time or day. Emphasizing this, Fig. 7 shows a four day time series comparison of three of the four models showing the diversity of the forecasts. This apparent lack of correlation between errors motivates the use of an ensemble forecasting approach [3], [27]: even the simple average of forecasts will provide a more accurate forecast than any one forecast on its own. Fig. 8 and Table I demonstrate the superior performance of ensemble forecasts comprising the ARIMA model (AR), the Gaussian Load Profile model (G), the Neural Network $(\mathrm{N})$ and the Persistent forecast.

Table I shows that this forecast is superior in most respects to its constituent forecast models although in the adjusted 4-norm it offers marginal advantages over the next best model, the Gaussian Load Profile on its own. Employing these models in practice may require scalability or reduced computing power (forecasting on the meter, a data concentrator or on a substation computer). Accordingly, Table II shows the corresponding computational effort required to make a forecast. The pre-trained ARIMA model, while a superior performer, takes significantly longer to forecast for the cohort of 123 premises than any other model. The Gaussian load profile and the Neural Network are roughly equivalent in terms of order of magnitude although the performance of the former model is superior. The flat and persistent forecasts are low computational effort, with the persistent forecast offering performance almost equivalent to that of the Neural Network at lower computational expense.

\section{CONCLUSION}

This paper has demonstrated several methods for forecasting residential electrical demand for small power systems which could allow more reliable scheduling of embedded generation permitting grid independence. Electrical networks that would benefit from progressively higher accuracy electrical demand predictions may be privately owned and with either network reinforcement constraints, community aspirations for sustainability or the desire to operate as an islanded power system. Although a challenging problem domain, without the supporting historical data that may accompany larger scale systems, it has indicated there are several algorithmic improvements for demand forecasting at the distribution and domestic LV networks that could be investigated to improve forecasting performance. As with HV/MV level forecasts, ARIMA models continued to offer high levels of forecasting accuracy albeit at higher computational expense. The Gaussian-Markov approach proposed here has been demonstrated to provide almost equivalent levels of accuracy to the ARIMA model but at lower computational expense, indicating that an on-meter solution could be a viable means of practical deployment. An ideal model would see the intra-day load profile characteristic encoding incorporated into the Gaussian model in a 'switching' type autoregressive model [28]. As more extensive and complete data sets become available, the day to day relationship between load profiles could be encoded as more complex mover-stayer [29] type models [30]. The diversity of forecasting techniques used motivated the investigation of an ensemble forecast using the models demonstrated - on the small data set presented this offered a significant reduction in both MAE and MAPE - if computation effort was no object, this solution would offer the highest accuracy. Further investigation in this field would be in the choice of constituent forecasts as well as the means of aggregating them - significant performance increase was attained through simple averaging but this could be further improved by weighting model outputs according to their time of day or day of week specialization.

\section{REFERENCES}

[1] Load Profiles and Their Use in Electricity Settlement. [Online]. Available: https://www.elexon.co.uk/wp-content/uploads/2013/11/ load_profiles_v2.0_cgi.pdf, accessed Nov. 3, 2015.

[2] J. Stephenson et al., "Energy cultures: A framework for understanding energy behaviours," Energy Policy, vol. 38, no. 10, pp. 6120-6129, 2010.

[3] T. Hong, P. Pinson, and S. Fan, "Global energy forecasting competition 2012," Int. J. Forecast., vol. 30, no. 2, pp. 357-363, 2014.

[4] M. Beccali, M. Cellura, V. Lo Brano, and A. Marvuglia, "Forecasting daily urban electric load profiles using artificial neural networks," Energy Convers. Manage., vol. 45, nos. 18-19, pp. 2879-2900, Nov. 2004.

[5] T. Hong, "Short term electric load forecasting," Ph.D. dissertation, Dept. Oper. Res. Elect. Eng., North Carolina State Univ., Raleigh, NC, USA, 2010. 
[6] N. Amjady, F. Keynia, and H. Zareipour, "Short-term load forecast of microgrids by a new bilevel prediction strategy," IEEE Trans. Smart Grid, vol. 1, no. 3, pp. 286-294, Dec. 2010.

[7] H. S. Hippert, C. E. Pedreira, and R. C. Souza, "Neural networks for short-term load forecasting: A review and evaluation," IEEE Trans. Power Syst., vol. 16, no. 1, pp. 44-55, Feb. 2001.

[8] A. Marinescu, C. Harris, I. Dusparic, S. Clarke, and V. Cahill, "Residential electrical demand forecasting in very small scale: An evaluation of forecasting methods," in Proc. 2nd Int. Workshop Softw. Eng. Challenges Smart Grid (SE4SG), San Francisco, CA, USA, May 2013, pp. 25-32.

[9] M. Rowe, T. Yunusov, S. Haben, W. Holderbaum, and B. Potter, "The real-time optimisation of DNO owned storage devices on the LV network for peak reduction," Energies, vol. 7, no. 6, pp. 3537-3560, 2014.

[10] J. Llanos, D. Suaez, R. Palma-Behnke, A. Nunez, and G. Jimenez-Estevez, "Load profile generator and load forecasting for a renewable based microgrid using self organizing maps and neural networks," in Proc. Int. Joint Conf. Neural Netw., Brisbane, QLD, Australia, 2012, pp. 1-8.

[11] J. Kwac, J. Flora, and R. Rajagopal, "Household energy consumption segmentation using hourly data," IEEE Trans. Smart Grid, vol. 5, no. 1, pp. 420-430, Jan. 2014

[12] F. L. Quilumba, W.-J. Lee, H. Huang, D. Y. Wang, and R. L. Szabados, "Using smart meter data to improve the accuracy of intraday load forecasting considering customer behavior similarities," IEEE Trans. Smart Grid, vol. 6, no. 2, pp. 911-918, Mar. 2015.

[13] J. E. Seem, "Pattern recognition algorithm for determining days of the week with similar energy consumption profiles," Energy Build., vol. 37, no. 2, pp. 127-139, 2005.

[14] H. Mori and T. Itagaki, "A precondition technique with reconstruction of data similarity based classification for short-term load forecasting," in Proc. IEEE Power Eng. Soc. Gen. Meeting, vol. 1. Denver, CO, USA, 2004, pp. 280-285.

[15] S. Valero et al., "Comparative analysis of self-organizing maps vs. multilayer perceptron neural networks for short-term load forecasting," in Proc. Int. Symp. Modern Elect. Power Syst. (MEPS), Wrocław, Poland, 2010, pp. $1-5$

[16] S. V. Verdu, M. O. Garcia, C. Senabre, A. G. Marin, and F. J. G. Franco, "Classification, filtering, and identification of electrical customer load patterns through the use of self-organizing maps," IEEE Trans. Power Syst., vol. 21, no. 4, pp. 1672-1682, Nov. 2006.

[17] S. M. Mousavi and H. A. Abyaneh, "Effect of load models on probabilistic characterization of aggregated load patterns," IEEE Trans. Power Syst., vol. 26, no. 2, pp. 811-819, May 2011.

[18] B. Stephen and S. J. Galloway, "Domestic load characterization through smart meter advance stratification," IEEE Trans. Smart Grid, vol. 3, no. 3, pp. 1571-1572, Sep. 2012.

[19] B. Stephen, A. J. Mutanen, S. Galloway, G. Burt, and P. Jarventausta, "Enhanced load profiling for residential customers," IEEE Trans. Power Del., vol. 29, no. 1, pp. 88-96, Feb. 2014.

[20] E. Shove, M. Pantzar, and M. Watson, The Dynamics of Social Practice. Newbury Park, CA, USA: Sage, 2012.

[21] K. Gram-Hanssen, "Standby consumption in households analyzed with a practice theory approach," J. Ind. Ecol., vol. 14, no. 1, pp. 150-165, 2010.

[22] S. Higginson, E. McKenna, and M. Thomson, "Can practice make perfect (models)? Incorporating social practice theory into quantitative energy demand models," in Proc. 3rd Behave Energy Conf. Paradigm Shift Energy Efficien. Energy Reduct. Soc. Change, Oxford, U.K., Sep. 2014, pp. 3-4 and 17.

[23] I. Richardson, M. Thomson, D. Infield, and C. Clifford, "Domestic electricity use: A high-resolution energy demand model," Energy Build., vol. 42, no. 10, pp. 1878-1887, 2010.

[24] M. Chaouch, "Clustering-based improvement of nonparametric functional time series forecasting: Application to intra-day household-level load curves," IEEE Trans. Smart Grid, vol. 5, no. 1, pp. 411-419, Jan. 2014

[25] R. E. Kass and A. E. Rafferty, "Bayes factors," J. Amer. Stat. Assoc., vol. 90, no. 430, pp. 773-795, Jun. 1995.

[26] S. Haben, J. Ward, D. V. Greetham, C. Singleton, and P. Grindrod, "A new error measure for forecasts of household-level, high resolution electrical energy consumption," Int. J. Forecast., vol. 30, no. 2, pp. 246-256, 2014.

[27] C. E. Borges, Y. K. Penya, and I. Fernandez, "Evaluating combined load forecasting in large power systems and smart grids," IEEE Trans. Ind. Informat., vol. 9, no. 3, pp. 1570-1577, Aug. 2013.
[28] G. Yu and C. Zhang, "Switching ARIMA model based forecasting for traffic flow," in Proc. IEEE Int. Conf. Acoust. Speech Signal Process. (ICASSP), vol. 2. Montreal, QC, Canada, 2004, pp. 429-432.

[29] H. Frydman and A. Kadam, "Estimation in the continuous time mover-stayer model with an application to bond ratings migration," Appl. Stoch. Models Bus. Ind., vol. 20, no. 2, pp. 155-170, 2004.

[30] L. Saul and F. Pereira, "Aggregate and mixed order Markov models," in Proc. 2nd Conf. Empir. Methods Nat. Lang. Process., 1997, pp. 81-89.

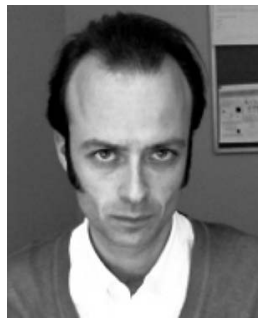

Bruce Stephen (M'09-SM'14) received the B.Sc. degree from Glasgow University, and the M.Sc. and Ph.D. degrees from the University of Strathclyde. $\mathrm{He}$ is currently a Senior Research Fellow with the Institute for Energy and Environment, University of Strathclyde. He is a Chartered Engineer. His research interests include distributed information systems, machine learning applications in power system, and energy end use.

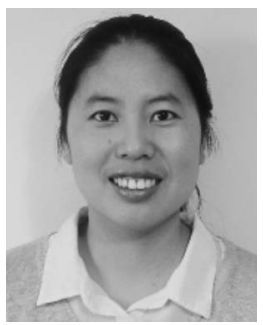

Xiaoqing Tang received the B.Eng. and M.Eng. degrees from the Harbin Institute of Technology, China, in 2010, and the Ph.D. degree from the Queen's University of Belfast, U.K., 2013. She is a Research Associate with the Institute for Energy and Environment, University of Strathclyde. Her research interests include demand and renewable generation modeling, and application of statistical techniques in power systems.

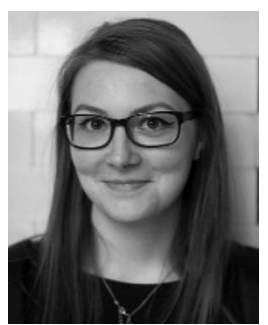

Poppy R. Harvey received the B.Eng. degree from the University of Strathclyde, Glasgow, U.K. She is currently a Research Assistant with the Institute for Energy and Environment, University of Strathclyde. Her research interests include appliance load disaggregation and modeling residential demand.

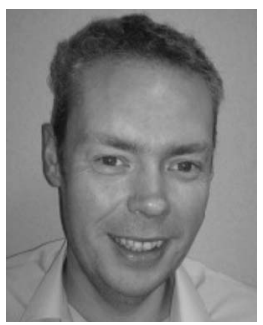

Stuart Galloway received the B.Sc. (Hons.) degree from the University of Paisley, and the M.Sc. and $\mathrm{Ph} . \mathrm{D}$. degrees in mathematics from the University of Edinburgh, in 1998. He is the Reader with the Institute for Energy and Environment, University of Strathclyde. His core research interests include aero, marine, and energy electrical activities, and strategic and applied research.

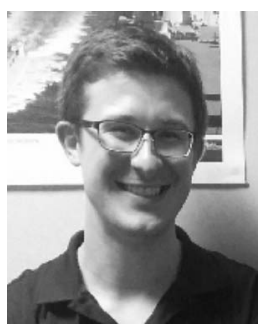

Kyle I. Jennett received the M.Eng. and Ph.D degrees from the University of Strathclyde, Glasgow, U.K. $\mathrm{He}$ is a Research Associate with the Institute for Energy and Environment, University of Strathclyde. His research interests include the implementation of future power systems encompassing DG, power system protection, and demand side management. 